\title{
Discriminative MR Image Feature Analysis for Automatic Schizophrenia and Alzheimer's Disease Classification ${ }^{\star}$
}

\author{
Yanxi Liu ${ }^{1 \star \star}$, Leonid Teverovskiy ${ }^{1}$, Owen Carmichael ${ }^{1}$, Ron Kikinis ${ }^{2}$, \\ Martha Shenton ${ }^{2}$, Cameron S. Carter ${ }^{3}$, V. Andrew Stenger ${ }^{4}$, Simon Davis ${ }^{4}$, \\ Howard Aizenstein ${ }^{4}$, James T. Becker ${ }^{4}$, Oscar L. Lopez ${ }^{4}$, and \\ Carolyn C. Meltzer ${ }^{4}$ \\ 1 Carnegie Mellon University \\ 2 Harvard Medical School \\ 3 University of California, Davis \\ 4 University of Pittsburgh
}

\begin{abstract}
We construct a computational framework for automatic central nervous system (CNS) disease discrimination using high resolution Magnetic Resonance Images (MRI) of human brains. More than 3000 MR image features are extracted, forming a high dimensional coarseto-fine hierarchical image description that quantifies brain asymmetry, texture and statistical properties in corresponding local regions of the brain. Discriminative image feature subspaces are computed, evaluated and selected automatically. Our initial experimental results show 100\% and $90 \%$ separability between chronicle schizophrenia (SZ) and first episode SZ versus their respective matched controls. Under the same computational framework, we also find higher than $95 \%$ separability among Alzheimer's Disease, mild cognitive impairment patients, and their matched controls. An average of $88 \%$ classification success rate is achieved using leave-one-out cross validation on five different well-chosen patient-control image sets of sizes from 15 to 27 subjects per disease class.
\end{abstract}

\section{Introduction}

Schizophrenia (SZ) is a severe, chronic and persistent mental disorder with onset in late adolescence or early adulthood resulting in lifelong mental, social and occupational disability. Alzheimer's Disease (AD) is a disease of aging, and the financial and social burdens of $\mathrm{AD}$ are compounded by recent and continued increases in the average life span. Assisting clinicians in making accurate early diagnostic distinctions for $\mathrm{SZ}$ and $\mathrm{AD}$ becomes increasingly important with the development of effective treatments for CNS diseases.

Structural Magnetic Resonance (MR) images have an important advantage over other imaging modalities in that they are non-invasive and provide detailed

\footnotetext{
* This work is supported in part by NIH grants AG05133 and DA015900-01.

** Corresponding Author: yanxi@cs.cmu.edu 

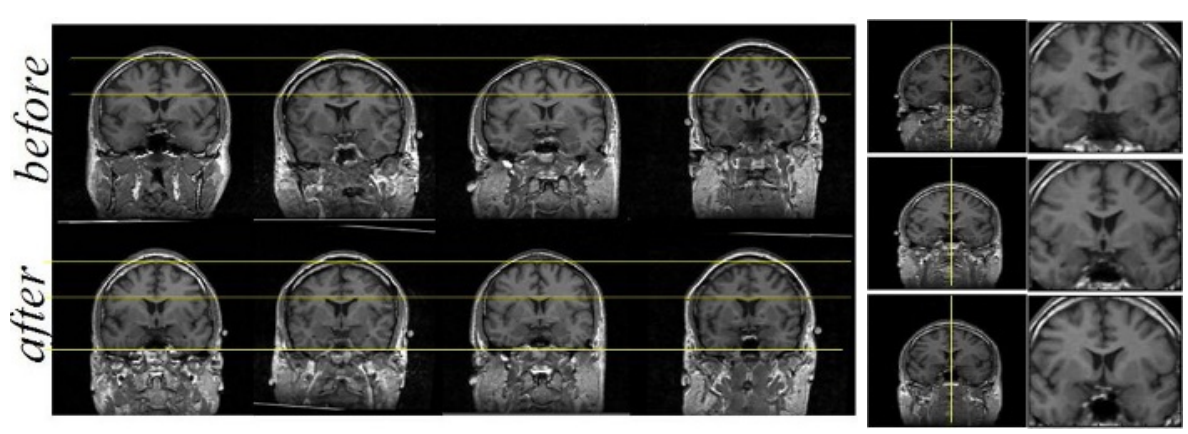

Fig. 1. Left: sample corresponding slices from four subjects (left to right: control, sz, control, sz). Before and after affine registration vertical correspondences are indicated by horizontal lines. Right: input consecutive slices of one subject where the iMSP is aligned with the center of the image and the ROI is cropped uniformly in preparation for brain asymmetry and other image-features computation.

information about gray and white matter parenchyma of the brain, and cerebrospinal fluid (CSF)-filled spaces. Nevertheless, classification of SZ and AD patients using high resolution MR neuroimages remains a challenging task even for the most experienced neuroradiologists. Most work in automatic or semiautomatic MR neuroimage classification [15/14,7!16] has been focusing on precise segmentation of various anatomical structures for volumetric and local shape comparisons 1416. In both cases of SZ and AD, there are considerable reported group morphological differences in specific anatomical structures of the brain [3] 18. Due to group overlap, however, few existing methods reliably distinguish whether an individual MR image is from a specific disease category (SZ vs. normal or AD vs. normal), particularly in early stages of the disease.

Section 2 gives a general description of our image-feature based classification approach. Section 3 describes our experiments on SZ and AD datasets. In Section 4 we discuss the results and summarize the paper. A more detailed report of this work can be found in 12 .

\section{General Approach}

We propose an image feature based statistical learning approach, in addition to anatomical morphology analysis, to better classify MR image of CNS diseases. We formulate this task as a supervised learning problem, where the MR image labels are given (class decisions are made by doctors based on specific clinical criteria for SZ and AD through behavior and cognitive tests). The key element is to learn those MR image features that best discriminate disease classes. We shall examine both separability on the training data to visualize the data distribution, and generality in terms of leave-one-out cross validation results to evaluate the predicting power of the selected MR image features. The basic components in our computational framework include: 
3D Image Alignment: All MR images for each classification problem in our feasibility study are taken using the same scanner and protocols. We verify image intensity consistency by carrying out an analysis of intensity histograms of all input images. All MR images are deformably registered using an affine registration algorithm 13 to a digital brain atlas (the Harvard brain atlas 9] for schizophrenia study and the Montreal Neurological Institute (MNI) template 4 for Alzheimer's Disease study). Affine deformable registrations normalize all brain images for shape and scale globally. Internal local differences are not further corrected intentionally.

An automatic ideal midsagittal plane (iMSP) extraction algorithm [1] is applied to each 3D MR image before and after the affine registration to (1) initialize yaw, roll angles and X-axis translation [11] before the 3D registration for faster convergence and better registration accuracy. (2) validate and reassure the iMSP accuracy after affine registration (Figure 1) in preparation for quantifiedbrain-asymmetry image feature extraction.

Regions of Interest: Since each brain image is affinely registered with a digital atlas in 3D space, our medical experts can identify a region of interest (ROI) by specifying a stack of $2 \mathrm{D}$ slices on the atlas. These are regions which may have potential relevance to disease classification on individual MR scans as indicated in the literature.
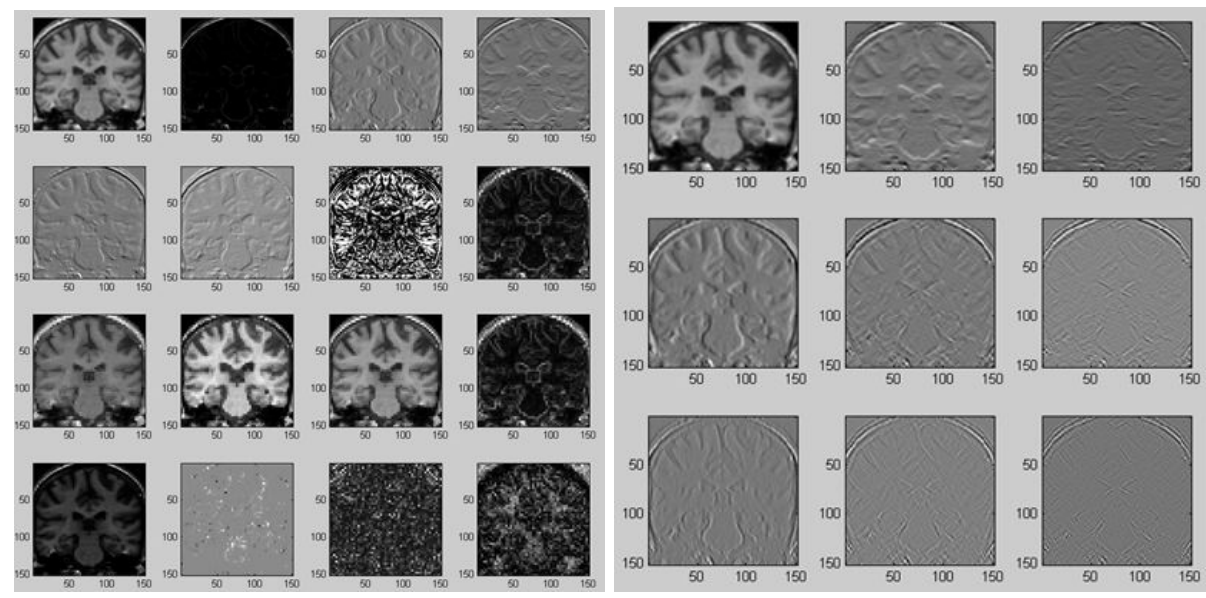

Fig. 2. Left: A sample view of all extracted statistical features. From left to right, top to bottom: mean intensity, variance, vertical edge, horizontal edge, diagonal edge, (other) diagonal edge, edge orientation, standard deviation, maximum intensity, minimum intensity, median intensity, range, energy, skewness, kurtosis, entropy. Right: the top-left 3 by $32 \mathrm{D}$ texture features out of the 25 Law's texture features - L5L5, E5L5, S5L5; L5E5, E5E5, S5E5: L5S5, E5S5, S5S5. The five 1D convolution kernels: Level, L5 = [ $\left.\begin{array}{lllll}1 & 4 & 6 & 4 & 1\end{array}\right]$; Edge, E5 = [ [-1 - $\left.\begin{array}{llll}-2 & 0 & 2 & 1\end{array}\right]$; Spot, S5 = [ $\left.\begin{array}{lllll}-1 & 0 & 2 & 0 & -1\end{array}\right]$; Wave, W5 = [ - $\begin{array}{ll}-1 & 2\end{array}$ 0 - -21 ] $]$; and Ripple, R5 = [ $\left.\begin{array}{lllll}1 & -4 & 6 & -4 & 1\end{array}\right]$. Each two-dimensional convolution kernel is generated by convolving a vertical $1 \mathrm{D}$ kernel with a horizontal $1 \mathrm{D}$ kernel. 


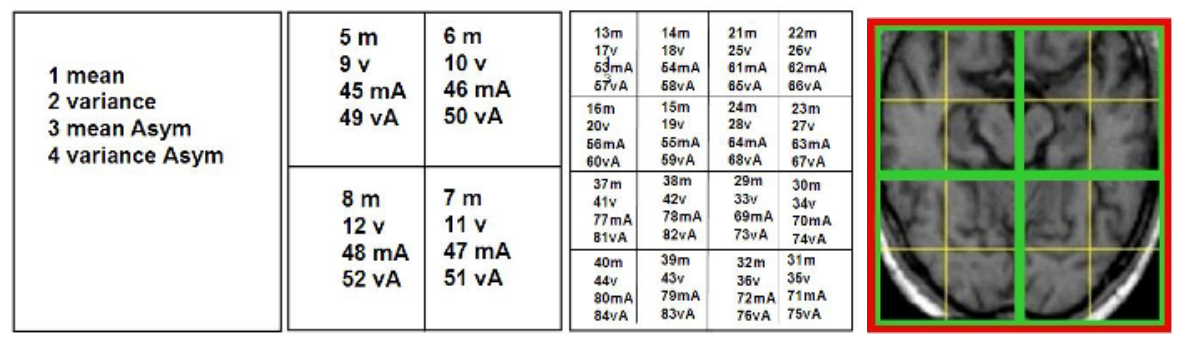

Fig. 3. The three left-panels illustrate the hierarchical decomposition of each slice. For each image feature, we compute the mean $(\mathrm{m})$, variance $(\mathrm{v})$, asymmetry mean $(\mathrm{mA})$, and asymmetry variance(vA) in each local region (right). We then concatenate all of these local measures into a feature vector. The numbers in each block indicate an index of the feature location (clockwise rotation of the four quarters starting from top-left).

Image Features: Two general categories of 41 image features are used in our feasibility study, including 16 statistical features [8] and 25 Law's texture features [10] (Figure 2).

For each filtered brain slice $I(x, y)$, we also compute an asymmetry brain image feature defined as: $D(x, y)=I(x, y)-I_{v R e f}(x, y)$ where $I_{v R e f}$ is the vertical reflection of the original feature image $I(x, y)$. Since $I(x, y)$ is already centered by the iMSP, $D(x, y)$ is the intensity difference of the corresponding left and right halves of a brain slice. Left-right asymmetry redundancy is removed during feature screening process.

Image Feature Location: One important aspect of our exploration is to localize where the potential discriminative features lie in the ROI. We subdivide each slice of the registered brain (in coronal or axial direction) hierarchically. Figure 3 shows such a division on three levels (each level has 1, 4 and 16 regions respectively). For each level, we compute the mean and variance of the image feature in each subdivision. Given both original image feature and bilateral asymmetry difference measures, a total of $(1+4+16) * 4=84$ "location features" are generated for each image feature type on each $2 \mathrm{D}$ slice. Therefore we have a total of $41 \times 84 \times \#$ of slices $=3444 \times \#$ of slices dimensional feature space with regional, asymmetry, statistical and textural information to explore.

Discriminative Feature Evaluation and Screening: A common theme in our research is to use available image features selectively for different image discrimination tasks; this is especially effective when redundancy exists among different feature dimensions which is highly characteristic in image feature-based classifications. We define an augmented variance ratio (AVR) as

$$
A V R(F)=\frac{\operatorname{Var}\left(S_{F}\right)}{\frac{1}{C} \sum_{i=1 . . C} \frac{\operatorname{Var}_{i}\left(S_{F}\right)}{\min _{i \neq j}\left(\left|\operatorname{mean}_{i}\left(S_{F}\right)-\operatorname{mean}_{j}\left(S_{F}\right)\right|\right)}}
$$

where $\operatorname{Var}\left(S_{F}\right)$ is the cross-class variance of feature $F, \operatorname{Var}_{i}\left(S_{F}\right)$ and $\operatorname{mean}_{i}\left(S_{F}\right)$ are the within-class variance and mean of feature $F$ for class $i$ out of $C$ distinct classes. Similar to Fisher criteria [6], AVR is the ratio of cross-class variance of 
the feature over within-class variance, with an added penalty to features that have close inter-class means. AVR ranked features provide us with a quantitative basis to screen out non-discriminative features before feature subset selection [2]. Feature subset selection is carried out using Linear Discriminant Analysis (LDA) 6] whose criteria is consistent with AVR.

Separability Analysis: We define separability of a given data set $D$ as the classification rate (plus sensitivity and specificity) in a learned discriminative feature subspace on $D$ using a K-nearest neighbor (KNN) classifier [6]. Different feature subspaces are explored using either an exhaustive search for all triplets or a forward sequential selection strategy [2]. The result is a set of image feature subspaces with the highest classification rates indicating best separation among image classes.

Prediction: Given $N$ data points (3D MR images from $\mathrm{N}$ different subjects), $N-1$ are used for training to find discriminative feature subspaces, and the one left out is used as the unseen test sample for evaluating the prediction accuracy of the learned classifier. This process is repeated $N$ times in a round-robin manner.

\section{$3 \quad$ Experiments}

\subsection{Classification of Schizophrenia Patients}

A feasibility study is carried out using (1) an image data set from Dr. Shenton [17. containing MR images of 15 schizophrenia patients (chronicle) and 15 controls; and (2) an image data set from Dr. Carte1 1 , containing MR images of 24 first episode (FE) schizophrenia patients and 27 normal controls. The controls are matched in age, family background and handedness. From each 3D MR scan a set of $2 \mathrm{D}$ coronal slices are sampled around the region of interest. Taking the top 30 most discriminative features from more than 3000 candidates, followed by sequential forward feature subset selection using LDA [2], and LOO using KNN we achieve the results listed in Table 4 and Figure 5.

\subsection{Classification of Alzheimer's Disease}

A set of 60 subjects are selected by experts from the Alzheimer's Disease Research Center (ADRC - an NIH research center) of University of Pittsburgh, in which 20 are normal controls, 20 are subjects with MCI, and 20 are diagnosed $\mathrm{AD}$ patients matched on age, education and sex. The image data are acquired on a $1.5 \mathrm{~T}$ GE scanner in the coronal plane, with minimized partial voluming effects. This image data set is normalized for intensity mean ( 0.4 in a 0 to 1 scale) and variance (0.25). See Table 1 and Figure 6 for classification results on this data set.

Combination of Image Features with Shape Features: Using LONI 15 we have hand-segmented hippocampi for each subject in the 20-20-20 (control, MCI and AD) image data set. Several shape features are computed using the hand

\footnotetext{
${ }^{1}$ originally from University of Pittsburgh, now at UC Davis
} 


\begin{tabular}{|l|c|c|}
\hline $\begin{array}{l}\text { Disease Class } \\
\text { \# of Subjects }\end{array}$ & $\begin{array}{c}\text { CTL vs. SZ } \\
\text { 15 vs. 15 }\end{array}$ & $\begin{array}{c}\text { 27 vs. SZ } \\
\text { vs. 24 }\end{array}$ \\
\hline Separability & $100 \%$ & $90 \%$ \\
\hline Sensitivity & $100 \%$ & $88 \%$ \\
\hline Specificity & $100 \%$ & $92 \%$ \\
\hline \hline LOO Rate & $90 \%$ & $78 \%$ \\
\hline Sensitivity & $93 \%$ & $79 \%$ \\
\hline Specificity & $87 \%$ & $77 \%$ \\
\hline
\end{tabular}

Fig. 4. Experimental Results: Harvard data set contains $15 \mathrm{SZ}$ patients (chronicle) versus 15 normals. UPMC data set contains $24 \mathrm{SZ}$ patients (first episode) versus 27 normals.
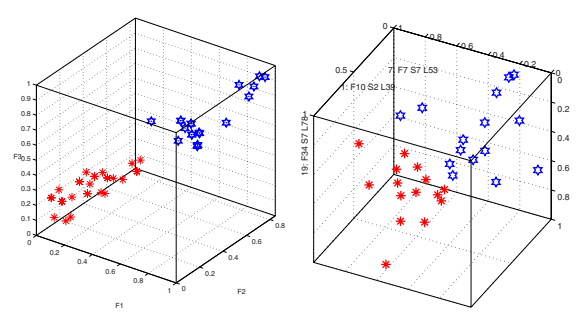

Fig. 5. Examples of two automatically selected 3-feature discriminative subspaces for schizophrenia MR image data sets (1) and (2). Stars (lower-left) indicate SZ patients.

Table 1. Alzheimer's Disease Classification Results

\begin{tabular}{|c|c|c|c|c|}
\hline $\begin{array}{c}\text { Disease Class } \\
\begin{array}{c}\text { Cof Subjects } \\
\text { Features Used }\end{array}\end{array}$ & $\begin{array}{c}\text { CTL vs. MCI } \\
\text { Image }\end{array}$ & $\begin{array}{c}\text { CTL vs. AD } \\
20 \text { vs. } 20 \\
\text { Image }\end{array}$ & $\begin{array}{c}\text { MCI vs. AD } \\
20 \text { vs. } 20 \\
\text { Image }\end{array}$ & $\begin{array}{c}\text { MCI vs. AD } \\
20 \text { vs. 20 } \\
\text { Image+Shape }\end{array}$ \\
\hline Separability & $100 \%$ & $96 \%$ & $97 \%$ & $98 \%$ \\
\hline Sensitivity & $100 \%$ & $95 \%$ & $100 \%$ & $100 \%$ \\
\hline Specificity & $100 \%$ & $96 \%$ & $95 \%$ & $95 \%$ \\
\hline LOO Rate & $93 \%$ & $93 \%$ & $78 \%$ & $88 \%$ \\
\hline Sensitivity & $100 \%$ & $85 \%$ & $80 \%$ & $85 \%$ \\
\hline Specificity & $85 \%$ & $100 \%$ & $75 \%$ & $90 \%$ \\
\hline
\end{tabular}

traced 3D surface information. They are: hippocampus volume, the coordinate of the centroid of the hippocampus, the $\mathrm{x}, \mathrm{y}$, and $\mathrm{z}$ dimensions of the bounding box around the hippocampus, the 2nd-order geometric moments of the hippocampus along three axes, and 2nd-order legendre moments of the hippocampus. Adding these shape features to the image feature selection process we have achieved better classification rates (right-most column in Table 1), indicating that the image intensity features and shape features complement each other.

Experiments with Multiple Classifiers: We have also experimented with many standard classifiers including decision trees, decision graphs, decision stumps, instance-based learning, naive Bayes and support vector machines (SVM) with or without bagging or stacking on the top 100 AVR ranked image features. We found the performance depends primarily on the image features used. Using the top 30 AVR ranked image features in combination with shape features, for example, decision stumps achieves the best classification rates for control versus AD, 90\% (sensitivity and specificity). 


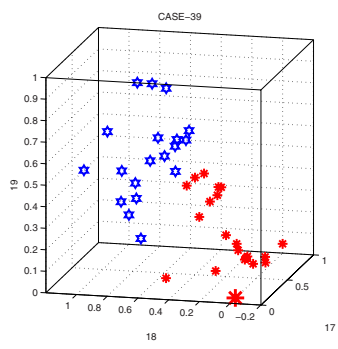

(1)

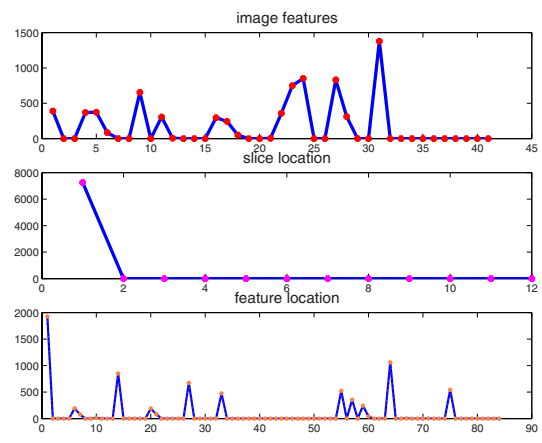

$(2)$

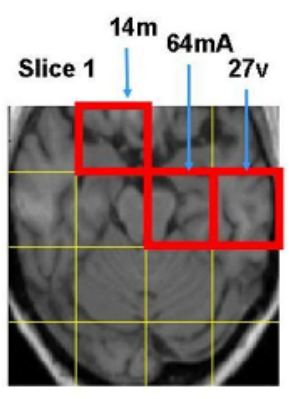

$(3)$

Fig. 6. (1): Sample LOO result showing the predicting power of the learned image feature space for MCIs versus normal controls. The larger star indicates the subject (an MCI patient) left-out during the training process. (2): Three plots showing feature type, slice number and geometric location of the most discriminative features selected for MCI vs. normal controls prediction. Top: The most popular image feature is the 15 th texture feature (31st on the $\mathrm{X}$ axis) composed of ripple and spot. Middle: The most important slice in a 3D image is the most inferior slice in the chosen ROI. Bottom: the top three most discriminative local features come from region 64 (mean intensity asymmetry value, hippocampus region), region 14 (mean value of the top left-middle region) and region 27 (the intensity variance). (3): the local regions containing the most discriminative local features are outlined on the most selected brain slice.

\section{Discussion and Conclusion}

We establish a framework for a computer system to automatically explore very high dimensional image feature spaces in search of a discriminative subspace. The preliminary results suggest that regional image features contain highly discriminative information to separate different CNS diseases, e.g. SZ or AD, from normal brains on several limited (15 to 27 subjects in each disease class) but well-chosen image sets. The LOO cross validation results also suggest the potential to build a disease predictor that uses critically chosen image features to classify an unknown individual image into one of the disease categories with probability much higher than chance. The LOO result of controls versus AD surpasses the result reported in [7, which is based on a one-time division of the input data. Our result, on the other hand, is based upon a statistically justified 40-way division of the input data [6]. Our result on SZ classification (Table 4) also surpasses the performance reported in [16] on the same image set.

One non-intuitive aspect of our approach perhaps lies in the fact that, contrary to most medical image analysis and particularly in SZ and AD MR image studies, no anatomical segmentation of the MR neuroimages is carried out. Instead, we bring all brain images into a common coordinate system where they are affinely registered and their iMSPs coincide, divide each $2 \mathrm{D}$ slice into equal sized geometric regions and compute image properties in each region where the 
true anatomical structures in corresponding patches may or may not correspond. Our method takes advantage of the intensity discrepancy caused by local noncorrespondences and examines quantitatively whether such discrepancies are representative of their image semantic class (disease) by statistical learning and cross validation. Another advantage of our method over, e.g. neural network approaches, is that it is not a black-box. We are able to visualize the relative weights in the found discriminative subspace, data points distributions, and trace back the type and the (anatomical) locations of selected image features (Figure 6).

The ultimate goal of our research is to understand the biological implications of the automatically selected discriminative features. Current results, considering local features from temporal lobe asymmetry (for SZ study) and hippocampus asymmetry (for AD/MCI study, Figure 6) highly discriminative, are encouraging. We plan to further validate the consistency of selected image features from LOO, explore the whole 3D brain systematically in future studies using volumetric image features, and test our method on larger MR image data sets.

\section{References}

1. R. Bilder, H. Wu, B. Bogerts, M. Ashtari, D. Robinson, M. Woerner, J. Lieberman, and G. Degreef. Cerebral volume asymmetries in schizophrenia and mood disorders: a quantitative magnetic resonance imaging study. International Journal of Psychophysiology, 34(3):197-205, December 1999.

2. C. M. Bishop. Neural Networks for Pattern Recognition. Clarendon Press, 1995. ISBN:0198538499.

3. R. Buchanan, K. Vladar, P. Barta, and G. Pearlson. Structural evaluation of the prefrontal cortex in schizophrenia. Am. J. of Psychiatry, 155:1049-55, 1998.

4. D. L. Collins, A. Zijdenbos, V. Kollokian, J. G. Sled, N. J. Kabani, C. J. Holmes, and A. C. Evans. Design and construction of a realistic digital brain phantom. IEEE Trans. Med. Imag., 17:463-468, 1998.

5. T. Crow. Schizophrenia as an anomaly of cerebral asymmetry. In K. Maurer, editor, Imaging of the brian in psychiatry and related fields. Springer-Verlag, 1993.

6. R. Duda, P. Hart, and D. Stork. Pattern Classification. John Wiley \& Sons, New York, 2001.

7. P. Freeborough and N. C. Fox. MR image texture analysis applied to the diagnosis and tracking of alzheimer's disease. IEEE Transactions on Medical Imaging, 17(3):475-479, June 1998.

8. A. Jain, R. Duin, and J. Mao. Statistical pattern recognition: a review. IEEE Trans. Pattern Analysis and Machine Intelligence, 22(1):4-37, Jan. 2000.

9. R. Kikinis, M. Shenton, D. Iosifescu, R. McCarley, P. Saiviroonporn, H. Hokama, A. Robatino, D. Metcalf, C. Wible, C. Portas, R. Donnino, and F. Jolesz. A digital brain atlas for surgical planning, model-driven segmentation, and teaching. IEEE Transactions on visualization and computer graphics, 2(3):232-240, Sept. 1996.

10. K. Law. Textured Image Segmentation. PhD thesis, University of Southern California, January 1980.

11. Y. Liu, R. Collins, and W. Rothfus. Robust Midsagittal Plane Extraction from Normal and Pathological 3D Neuroradiology Images. IEEE Transactions on Medical Imaging, 20(3):175-192, March 2001. 
12. Y. Liu, L. Teverovskiy, O. Carmichael, R. Kikinis, M. Shenton, C. Carter, V. Stenger, S. Davis, H. Aizenstein, J. Becker, O. Lopez, and M. Meltzer. Discriminative $\mathrm{mr}$ image feature analysis for automatic schizophrenia and alzheimer's disease classification. Technical Report CMU-RI-TR-04-15, The Robotics Institute, Carnegie Mellon University, Pittsburgh, PA, 2004.

13. F. Maes, A. Collignon, D. Vandermeulun, G. Marchal, and P. Suetens. Multimodality image registration by maximization of mutual information. IEEE Transactions on Medical Imaging, 16(2):187,198, 1997.

14. R. McCarley, C. Wible, Y. Frumin, J. Levitt, I. Fischer, and M. Shenton. MRI anatomy of schizophrenia. Society of Biological Psychiatry, 45:1099-1119, 1999.

15. D. Rex, J. Ma, and A. Toga. The LONI pipeline processing environment. Neuroimage, 19(3):1033-48, 2003.

16. M. Shenton, G. Gerig, R. McCarley, G. Szekely, and R. Kikinis. Amygdalahippocampal shape differences in schizophrenia: the application of $3 \mathrm{~d}$ shape models to volumetric mr data. Psychiatry Research Neuroimaging, 115:15-35, 2002.

17. M. Shenton, R. Kikinis, F. Jolesz, S. Pollak, M. Lemay, C. Wible, H. Hokama, J. Martin, B. Metcalf, M. Coleman, M. A. Robert, and T. McCarley. Abnormalities of the left temporal lobe in schizophrenia. response to roth, pfefferbaum and to klimke and knecht. New England Journal of Medicine, 327:75-84, 1992.

18. P. Thompson, D. MacDonald, M. Mega, C. Holmes, A. Evans, and A. Toga. Detection and mapping of abnormal brain structure with a probabilistic atlas of cortical surfaces. Journal of Computer Assisted Tomography, 21(4):567-81, 1997. 